\title{
Care Transitions: A Leverage Point for Safe and Effective Medication Use in Older Adults - A Mini-Review
}

\author{
Amanda S. Mixon ${ }^{a, b}$ Erin Neal ${ }^{c}$ Susan Bell ${ }^{d, e}$ James S. Powers ${ }^{a, e}$ \\ Sunil Kripalani ${ }^{b, e, f}$ \\ ${ }^{a}$ Department of Veterans Affairs, Tennessee Valley Healthcare System Geriatric Research Education and Clinical \\ Center (GRECC), and b Section of Hospital Medicine, Division of General Internal Medicine and Public Health and \\ 'Department of Pharmaceutical Services, d Division of Cardiovascular Medicine, Department of Medicine, Vanderbilt \\ University Medical Center, ${ }^{e}$ Center for Quality Aging and ${ }^{f}$ Center for Clinical Quality and Implementation Research, \\ Vanderbilt University, Nashville, Tenn., USA
}

\section{Key Words}

Medication adherence $\cdot$ Care transitions $\cdot$ Older adults

\begin{abstract}
Older adults often face challenges as they transition out of the acute care hospital, especially with regard to adhering to their medications. In this narrative review, we discuss medication adherence in older adults across the continuum of care, describing reasons for nonadherence, methods to assess adherence and tools to improve adherence, with particular focus on emerging techniques and technologies. Taking steps at care transitions to assess medications and foster adherence to the medication regimen can increase the safety of older adults following hospitalization.
\end{abstract}

(C) 2014 S. Karger AG, Basel

\section{Introduction}

Older adults typically take more medications than younger adults due to multimorbidity associated with advanced age. Adherence is defined as the extent to which an individual's medication-taking behavior follows the recommendations from a health care provider. Adherence incorporates filling or refilling medications as well as taking medications correctly, which in turn requires clarity on which medications to take and how to take them. Nonadherence significantly affects patient outcomes and the cost of health care. In a sample of more than 17,000 noninstitutionalized individuals aged 65 years and older in the USA, $40 \%$ reported some type of nonadherence [1]. Though age alone is not a risk factor for nonadherence, older adults are vulnerable to medication nonadherence issues, particularly during care transitions.

Care transitions occur when an older adult moves between different health care settings across the continuum of care. Of particular interest are the transitions surrounding a hospital stay, specifically the following: emergency department visits, hospitalization, discharge to home, post-acute care (PAC), home health, and outpatient follow-up in primary care and subspecialty clinics. During these interactions medications are frequently stopped, adjusted or newly prescribed. Differences between the medication lists, or discrepancies, are a common cause for confusion about the intended medication regimen and can lead to nonadherence as well as adverse drug events or harm due to medications. In the period

\section{KARGER 125}

C 2014 S. Karger AG, Base

$0304-324 \mathrm{X} / 14 / 0611-0032 \$ 39.50 / 0$

E-Mail karger@karger.com

www.karger.com/ger
Amanda S. Mixon, MD, MS, MSPH

Department of Veterans Affairs, Tennessee Valley Healthcare System Geriatric Research Education and Clinical Center (GRECC), Suite 6000 Medical Center East - North Tower 1215 21st Ave. South, Nashville, TN 37232-8300 (USA)

E-Mail Amanda.S.Mixon@ Vanderbilt.Edu 
following hospitalization $24 \%$ of older adults do not fill new prescriptions, $14 \%$ experience medication discrepancies and $46 \%$ are nonadherent to medications, reinforcing the fact that such transitions are an ideal time to improve adherence for older adults [2-4].

Though many reviews have been written on medication adherence, few have focused on care transitions and novel ways to improve adherence such as new technologies. This article discusses medication adherence across the continuum of care, focusing on care transitions such as those surrounding hospitalization, for adults aged 65 years and older. We discuss reasons for nonadherence, methods to assess adherence and tools to improve adherence, with particular focus on emerging techniques and technologies. The ensuing discussion is based primarily on studies conducted in the USA, where we practice, and is intended to contribute to the international dialogue on adherence.

\section{Adherence during the Transition out of the Hospital}

Transitions of care surrounding a hospitalization represent a vulnerable time for older adults. While only $8 \%$ of older adults in the USA are hospitalized annually, they frequently experience multiple care transitions after hospitalization [5]. With each care transition, older adults often face new health information and recommendations, changes in medication doses and frequencies and poor communication with health care providers. Therefore, all hospitalizations give health care providers a golden opportunity to review medications, assess adherence and impact a patient's adherence going forward.

During care transitions, health care providers are required to complete medication reconciliation, the process by which a provider 'compares the medications a patient should be using (and is actually using) to the new medications that are ordered for the patient and resolves any discrepancies' [6]. However, the process of medication reconciliation is error-prone. Over a third of patients have at least 1 discrepancy in the admission medication list, with most due to an inaccurate medication history. At hospital discharge, the erroneous and incomplete information from admission is often fed forward, coupled also with changes to the regimen or new medications to treat the acute illness. On average, an older adult being discharged from hospital has 2 new medications (range 1-13), 0.8 medications discontinued (range 1-9), 0.7 medications changed in frequency (range 1-6), and 0.5 medications changed in dose (range 1-3) [7].

Medication Adherence in Older Adults
Following hospitalization, over one quarter of older adults are discharged to a post-acute care (PAC) facility that provides nursing or rehabilitation therapy before returning home. The transfer of information from the acute hospital to the PAC is often disjointed and confusing for the providers at the $\mathrm{PAC}$, who frequently receive more than one version of the discharge medication list. With multiple lists, the risk of medication discrepancies increases, and the PAC provider often cannot distinguish the 'correct' medication list. A majority of patients (71\%) admitted to PAC have at least 1 medication discrepancy, and each has an average of 3.5 discrepancies, mostly in cardiovascular agents, opioids, neuropsychiatric agents, diabetes agents, antibiotics, and anticoagulants [8]. Inadvertently, then, the administration of medications in the PAC may not be adherent to the medication regimen ordered at hospital discharge because of poorly performed medication reconciliation and communication at hospital discharge. Suboptimal medication management is one reason why $19-24 \%$ of patients discharged to PAC are readmitted to the hospital within 30 days, resulting in costs exceeding USD 4 billion to Medicare [9]. Moreover, the risk of not filling new prescriptions for patients discharged from PAC is twice that of patients discharged home from acute hospitals [3]. Clearly, the dual transition - from hospital to PAC and then to home - doubles the risk of medication discrepancies, failure to fill new medications and poor communication, again potentially reducing adherence to the intended regimen.

Among older adults discharged from the hospital to home, $16 \%$ are referred to home health services to receive physical and occupational therapy and/or for skilled nursing services including medication management [10]. Medication discrepancies can be as high as $94 \%$ in this setting [11]. On average, home health nurses identify 3.3 discrepancies per patient, often involving insulin, anticoagulants, aspirin, and opioids - medications which cause adverse drug events and result in emergency department visits and readmissions [11]. Home health care could be an ideal setting for identifying and reconciling medication discrepancies soon after discharge in order to enhance adherence to the intended regimen. However, procedures for performing medication reconciliation across 14,000 home health care agencies are not standardized [12].

In summary, the errors in the discharge medication list from the hospital or PAC and inadequate counseling about medications all contribute to confusion about what older adults should be taking following hospitalization. As a result, older adults often inappropriately discontinue 
Table 1. Reasons for nonadherence to medication $[14,15]$

\begin{tabular}{|c|c|c|}
\hline Patient-related factors & $\begin{array}{l}\text { Forgetfulness } \\
\text { Did not want to take it } \\
\text { Lack of perceived need for medication } \\
\text { Physical/functional impairments } \\
\text { Lack of social support } \\
\text { Low health literacy } \\
\text { Low self-efficacy } \\
\text { Cost of medication } \\
\text { Transportation to get prescriptions } \\
\text { Physiological changes due to aging } \\
\text { Age-related decline in prospective memory }\end{array}$ & $\begin{array}{l}\text { (1) Patient did not fill new prescription } \\
\text { because it was too expensive (cost) } \\
\text { (2) Patient did not think antihypertensive } \\
\text { medications helped because s/he did } \\
\text { not feel differently when blood pressure } \\
\text { was elevated (lack of perceived need for } \\
\text { medication) }\end{array}$ \\
\hline $\begin{array}{l}\text { Provider and system } \\
\text { level factors }\end{array}$ & $\begin{array}{l}\text { Omission } \\
\text { Duplication } \\
\text { Inappropriate underuse } \\
\text { Incorrect dose or quantity } \\
\text { Polypharmacy and medication appropriateness } \\
\text { Multiple prescribing providers } \\
\text { Weakness in educating patients about discharge instructions } \\
\text { Unrecognized impairments in patients' cognition, physical } \\
\text { function, or health literacy }\end{array}$ & $\begin{array}{l}\text { (1) Physician increased dose of medication } \\
\text { at discharge, but did not give patient } \\
\text { new prescription for higher dose } \\
\text { (incorrect dose) } \\
\text { (2) Patient prescribed furosemide (generic } \\
\text { name) at discharge but already takes } \\
\text { Lasix (trade name; duplication, } \\
\text { unrecognized low health literacy) }\end{array}$ \\
\hline $\begin{array}{l}\text { Medication-related } \\
\text { factors }\end{array}$ & $\begin{array}{l}\text { Regimen complexity } \\
\text { Side effects } \\
\text { Unclear duration of treatment }\end{array}$ & $\begin{array}{l}\text { (1) Patient stopped medication because it } \\
\text { was causing diarrhea (side effect) }\end{array}$ \\
\hline
\end{tabular}

medications, restart home medications that were meant to be stopped, or inadvertently make mistakes with dosing and frequency. All forms of medication mismanagement ultimately reduce adherence to the intended regimen and increase the risk of patient harm such as adverse drug events. Nonadherence is also a significant contributor to unplanned health care utilization such as emergency department visits and hospital admission, as well as higher costs of care [13].

\section{Reasons for Nonadherence Surrounding Care Transitions}

The reasons underlying nonadherence to the medication regimen surrounding care transitions are complex but in general can be classified as factors related to patients, providers or health care systems, or the medications themselves (table 1). These categories are not mutually exclusive. For example, while forgetfulness or barriers to access the medication are generally categorized as patient-related, the misunderstanding of medication instructions is a result of ineffective communication be- tween health care providers and patients, as well as a system that often fails to provide patient-centered instructions. Cost is another barrier that could be categorized as related both to patients and to providers and health care systems. For example, older adults who have limited funds may find it difficult to pay for expensive prescriptions. Likewise, providers may not have an appreciation of how much certain medications cost, prescribing more expensive versions rather than an equally effective generic medication. It is important to acknowledge that in many instances nonadherence is not the patient's fault but rather reflects complex circumstances. Below, we discuss selected reasons for nonadherence in greater detail as they relate to care transitions.

Underlying these reasons for nonadherence are other risk factors such as living alone, an increasing number of chronic medical conditions, understanding the potential for adverse reactions, and utilizing multiple pharmacies [14-16]. At the end of a hospitalization, these risk factors may change as a result of alterations in an older adult's functional status, cognitive abilities and medication regimen. Therefore, the older adult's likelihood of nonadherence may increase after hospitalization. 


\section{Patient-Related Factors}

\section{Physical Challenges}

There are numerous physical factors that may affect the ability of an older adult to adhere to a medication regimen. After hospitalization, $40 \%$ of older adults experience greater dependency in activities of daily living which may impact medication self-administration [17]. Physical limitations may prevent older adults from getting to the pharmacy to fill a prescription and storing the medications appropriately. Furthermore, an older adult's ability to open medication bottles and take medications safely is impacted by their degree of visual impairment (prevalence of 30\%) [18], arthritis (prevalence of 50\%) which impacts manual dexterity [19] or impaired cognition which affects up to $32 \%$ of older adults following hospitalization [20]. Additionally, prospective memory (remembering to perform a certain event at a specific time in the future) also influences medication adherence, but it can vary widely across age groups of older adults and may depend on which tasks are involved [21,22].

\section{Health Literacy}

A factor that may be under-recognized in its impact on care transitions is health literacy, which is defined as 'the degree to which individuals have the capacity to obtain, process and understand basic health information and services needed to make appropriate health decisions' [23]. Advanced age is a risk factor for low health literacy; $29 \%$ of older adults have inadequate health literacy skills [24]. Low health literacy has been associated with difficulty understanding instructions and maintaining adherence following hospital discharge.

\section{Challenges due to the Processes of Aging}

Older adults are more likely to experience complications and side effects from commonly prescribed medications, which may affect adherence. Medications at doses suitable for younger adults may lead to unforeseen complications due to pharmacokinetic changes that occur with aging; this is particularly important for medications that have a narrow therapeutic window such as digoxin, antiepileptics, immune suppressants and some antibiotics [25]. The availability of a drug may be altered through volume of distribution (reduction in plasma protein levels, lean body mass, fat and total body water with age), resulting in highly protein-bound drugs with a greater free and active concentration, bioavailability and renal or hepatic clearance - the latter of which may have changed during an acute illness [25-27]. For hepatic clearance

Medication Adherence in Older Adults drugs the effects of a decrease in liver mass and hepatic blood flow along with age-related changes in activity of metabolizing enzymes suggest dosages should be reduced by up to $40 \%$ and renal-cleared drugs should be dosed according to the decline in glomerular filtration rate that occurs with each decade after 30 years of age $[26,27]$. Additionally, changes in pharmacodynamics in older adults can contribute to medication side effects. This relates to the varying effect that the same blood level of a drug has on different people and is most frequently seen with sedative or opioid medications where dosing is less predictable and which result in increased sedation with lower concentrations [28]. The prevalence of side effects may also be affected by drug-drug interactions and drug-disease interactions [29]. A drug's effect can be altered by competitive displacement from binding sites, antagonistic actions, inhibition or activation of hepatic enzymes, or synergistic side effects such as QT prolongation with antibiotics and antidepressant therapies [30]. For older adults the impact of changing and slowing reflexes and adrenergic and parasympathetic systems results in a higher likelihood of side effects [31]. Furthermore, as new medications are initiated or existing medications changed during hospitalization, side effects may be a more prominent problem in care transitions, potentially leading to nonadherence due to premature discontinuation of medications.

\section{Cost}

Medication costs contribute significantly to nonadherence in older adults [32]. In 2011 over 3.6 million older Americans were below the poverty line, with a further 2.4 million classified as near poverty [33]. Paying for brand name medications with high copayments and even generic medications can be a financial strain. For example, before the implementation of Medicare Part D (prescription drug insurance) in the USA, 2 million older adults were nonadherent to their drug regimen due to lack of prescription coverage [34]. Despite the improvements resulting from Medicare Part D, obstacles remain. The design of Medicare Part D includes a coverage gap, or 'donut hole' that requires beneficiaries to pay $100 \%$ of drug costs after exceeding an annual threshold. Research has shown that the 'donut hole' increased nonadherence, with fewer prescriptions filled and more gaps in therapy [35]. The Medicare Part D coverage gap should be phased out by 2020 and will benefit older adults facing financial barriers. In the interim, it is especially important to assess financial barriers at each care transition by asking questions such as, 'How often do you miss your medicines 
because you can't afford them?', or 'How do you pay for your medicines?' In addition, generics and lower cost alternatives should be prescribed when available. When out-of-pocket expenses are reduced, medication adherence improves for several chronic diseases.

Despite the link between adherence and cost of therapy, efforts to reduce or eliminate copayments have had an inconsistent effect on adherence rates [36]. Furthermore, it is clear that countries that subsidize the cost of medications also have significant issues with adherence, which reinforces the need to address additional factors through a multifaceted approach [37].

\section{Provider and System Level Factors}

\section{Polypharmacy}

Polypharmacy, defined as taking $\geq 4$ chronic medications, is associated with nonadherence as well as inappropriate prescribing, drug interactions, adverse drug events, hospitalization, and mortality. Often, the standard of care for individual chronic illnesses leads to polypharmacy and potential drug-drug interactions for individuals with multiple chronic illnesses. Health care providers need to prioritize the therapies to act in the older adult's best interest, eliminating medications that are not clinically indicated. Studies have estimated that $50 \%$ of older adults are taking at least 1 medication with no ongoing indication, and many of these drugs are initiated during hospitalization such as stress ulcer prophylaxis and antipsychotics for delirium [38]. Medication reviews should be performed at every transition of care to assess for and reduce unnecessary medications. All of the medications that a patient is taking should be reviewed, including over-the-counter medications and supplements that may introduce additional cost and complexity to the regimen with perhaps no therapeutic benefit. Ensuring patients are on as few medicines as necessary to manage their diseases will improve adherence and patient safety and reduce medication costs for the patient.

\section{Under-Treatment, Over-Treatment, and Medication}

\section{Appropriateness}

While polypharmacy is a well-documented issue that predisposes older adults to adverse drug events and nonadherence, under-treatment of conditions is also a problem. Not prescribing a potentially beneficial medication when clinically indicated is considered an error of omission, and indicates a provider and system level problem in evidence-based support for prescribing. There are errors of omission in over half of hospitalized elderly patients, and the probability of having an omission increases with advanced age and being female [39]. The most common prescribing omissions described were statins for the treatment of atherosclerotic disease, warfarin for chronic atrial fibrillation, ACE inhibitors for chronic heart failure, and antiplatelet therapy for arterial disease [39].

The Beers Criteria and the STOPP/START criteria can be used to detect inappropriate overuse or underuse of medication $[40,41]$. These explicit screening tools are intended to alert the provider to particular medications that are potentially inappropriate based on the patient's diagnoses, but are not a substitute for clinical judgment that incorporates the patient's preferences. Risk-benefit should be discussed with the patient and caregiver each time a medication is added to the patient's regimen, congruent with the patient's goals of care. This concern becomes even more important with multimorbidity [42]. The medication list should also be reviewed for appropriateness at each care transition.

\section{Medication-Related Factors}

\section{Regimen Complexity}

The complexity of the medication regimen is inversely associated with medication adherence. Many factors contribute to regimen complexity, including the number of medications, the number of trips to the pharmacy for unsynchronized refills, the frequency of administration, special medication instructions, and variability in dosage forms. Research shows that the rate of adherence significantly decreases when the frequency changes from onceto thrice-daily dosing [43]. For example, while there is no statistically significant difference in adherence between once-daily (79\%) and twice-daily (69\%) dosing, increasing frequency to 3 and 4 times a day results in much lower adherence rates of 65 and 51\%, respectively [43]. Special medication instructions, such as alternating daily doses for warfarin or crushing a tablet before administration, also increase regimen complexity and could impact adherence, especially in older adults with low health literacy. Other routes of administration like eye drops or inhalers can also present issues for older adults because of reduced dexterity and more difficulty coordinating the delivery of the drug. Using long-acting formulations when available and implementing a universal medication schedule by tailoring medications to be taken at set times 
of the day (morning, noon, evening, and night) can help decrease the regimen complexity and limit the number of administration times per day. Another way to decrease complexity is to synchronize fill dates at the pharmacy so all medications can be picked up at the same time.

\section{Assessing Adherence}

Though an older adult may have many reasons for nonadherence, the actual degree of adherence may not be evident unless it is specifically assessed.

\section{Objective Approaches}

Objective methods include performing pill counts, measuring medication concentrations in blood samples, using electronic monitoring systems, and obtaining prescription refill or claims data. These methods do not reveal the reasons for nonadherence and, with the exception of pill counts, they require specialized resources to implement. Moreover, although these methods are considered objective, they may actually provide inaccurate or unreliable information in certain situations. For example, pill counts and measuring blood concentrations may overestimate adherence because patients may 'dump pills', adjusting the number of medications in their bottles prior to assessment, or appear to have adequate levels because they took medications in the period leading up to assessment. Electronic monitoring systems may underestimate adherence if a patient removes more than 1 tablet at a time (such as to fill a pill box), or overestimate adherence if they open the container without removing medication.

Prescription fill and claims data can be used to assess adherence on a patient or population level. The most common methods to report adherence include the Medication Possession Ratio (MPR), Proportion of Days Covered (PDC), and the Cumulative Measure of Medication Gaps (CMG). These methods measure the day supply that a patient fills within an established time frame (MPR, PDC) or the gaps in time between refills (CMG) [44]. These data can demonstrate whether a patient has filled their medications, but will not establish that the patient is actually taking the medication. Additionally, claims data will not capture 'cash' purchases, which are often used for discounted generics and over-the-counter medicines. Using this information in a population of older adults transitioning between facilities can also be challenging because patients may fill their prescriptions at different pharmacies for which data are not linked.

Medication Adherence in Older Adults
Table 2. Questions to ask patients regarding medication adherence

Tell me the medicines you take and how you take them.

How often do you forget to take your medicine?

Most patients have trouble taking their medicines right on time every day; in the last week, how many times have you missed a dose of a medicine?

Have you ever cut back or stopped taking your medicine without telling your doctor because you felt worse with it, felt like your symptoms were under control or had trouble paying for it?

When was your last dose of this medicine?

Taking medication every day can be very inconvenient; how inconvenient is it for you to take your medicine like it is ordered for you?

In the last year, what's the longest time you have gone without one of your medicines?

What is the longest time you have gone between running out of your medicine and getting it refilled?

\section{Patient-Centered Approaches}

Direct questioning of the patient or caregiver is a subjective but simple way of assessing adherence. No specialized resources are required and, by elucidating reasons for nonadherence, questioning may yield the most beneficial and actionable information. Having a conversation using open-ended, nonjudgmental questions - i.e. 'Tell me how you take your medicines', or 'Most patients miss their medicines from time to time. How often do you miss a dose of your medicine?' - will help reveal the patient's medication-taking behaviors. It is important, though, not to ask a single question in a cursory manner, but rather to initiate a discussion that includes follow-up prompts to elicit more details of the patient's medication use patterns (table 2).

The conversation will help assess the patient's adherence and will provide clues about medication understanding, patient perceptions and beliefs, and barriers to taking medication as prescribed. Several formal assessment tools such as the DRUGS tool [45], the MedTake tool [46] and the Adherence to Refills and Medications Scale [47] quantify adherence and the ability to manage medications in a systematic way. Adherence measured by self-report and monitoring systems are moderately correlated. Furthermore, self-reported adherence predicts clinical outcomes such as hospitalization. A busy health care provider, therefore, can adequately capture adherence using simple self-reported measures [48, 49]. 
Table 3. Tools to address medication adherence

\begin{tabular}{ll}
\hline Category & Tool \\
\hline $\begin{array}{l}\text { Medication } \\
\text { lists }\end{array}$ & $\begin{array}{l}\text { Conventional typed or hand-written list } \\
\text { Illustrated pill cards/charts }\end{array}$ \\
\hline Reminders & $\begin{array}{l}\text { Traditional - charts, calendars, phone calls, } \\
\text { postcards } \\
\text { Electronic - e-mail, text messaging, electronic } \\
\text { reminder device }\end{array}$ \\
\hline Dispensers & $\begin{array}{l}\text { Calendar pill organizers (pill box) } \\
\text { Calendar blister packaging }\end{array}$ \\
& Electronic dispenser \\
\hline $\begin{array}{l}\text { Real-time, } \\
\text { multimodal }\end{array}$ & $\begin{array}{l}\text { Electronic calendar pill organizers with remote } \\
\text { monitoring and feedback }\end{array}$ \\
tools & $\begin{array}{l}\text { Electronic reminder device with remote } \\
\text { monitoring and feedback }\end{array}$ \\
\hline
\end{tabular}

Recognizing nonadherence is only the first step. The provider must also assess why the patient's medicationtaking behavior does not match the intended therapy. Identifying and addressing barriers in a patient-centered way may provide an opportunity to clarify misunderstanding about the medication regimen, simplify the schedule or eliminate a drug that is expensive or causing side effects. Frequently, these tasks are completed by primary care providers, who play a critical role in medication management across the continuum of care.

\section{Tools to Address Adherence}

Numerous interventions have been developed and tested to improve medication adherence, including patient-centered education, behavioral support, case management, collaborative care, and care coordination [50]. Here, however, we focus on tangible tools that range from classic to new technologies (table 3). Although the quality of the evidence is low in general, we sought to summarize what is available, either from previous reviews or primary literature.

National organizations recommend that older adults use a standardized form to keep an updated list of their medications and update it at each care transition. Providing patients with an updated medication list that incorporates illustrations for the medication's purpose and dosing times seems to offer additional benefit, improving comprehension, recall and adherence, especially in populations with lower health literacy [51].
Traditional reminders in the form of charts, calendars, phone calls, or postcards may have a small effect on adherence in older adults. Additionally, electronic reminders - by electronic mail, text messaging or electronic reminder devices - generally improve short-term adherence. In recent systematic reviews, short message service reminders like text messages may increase adherence $[52,53]$. Few studies have targeted older adults for testing e-mail or text message reminders $[54,55]$. Electronic reminder devices, such as caps that affix to prescription bottles and indicate when the last dose of medication was taken, have been tested in uncontrolled studies, and no conclusions should be drawn about their efficacy.

Dispensing tools range from calendar pill organizers (e.g. weekly pill box) and calendar blister packaging (i.e. pills for the patient grouped in a sealed and dated container) to an automated dispenser of all medications at a given administration time, as seen in hospitals. Calendar pill organizers and calendar blister packaging may have a positive effect on adherence, but a review by Zedler et al. [56] noted significant methodological limitations related to sample size, study duration and differing outcomes of the 10 studies included. Electronic chip technology has been integrated into calendar blister packaging, but more feasibility testing is needed. Lastly, devices that essentially dispense a patient's medications at the correct time have been piloted, but controlled studies are needed [57].

Multimodal solutions integrate tools discussed above with remote monitoring and feedback capabilities. Caps exist that affix to prescription bottles, turn colors to indicate the time for an individual's next dose and contact someone if that dose is not taken. Additionally, an electronic pill organizer has been fused with a flatbed scanner which sends a digital image of remaining pills for distance monitoring and provides phone support. Despite anecdotal benefit, we were unable to find any evidence from controlled studies for the effectiveness of such multimodal solutions, but this work may be underway.

Finally, the tools to improve medication adherence may be combined for synergistic effects. In the spirit of patient-centered care, however, the choice of adherence tool(s) must be customized to address the older adult's reasons for nonadherence. Moreover, there are very few studies comparing tools directly to each other or examining their cost-benefit or cost-effectiveness. Larger, welldesigned trials are needed to examine the effects of adherence tools, particularly in the time following hospital- 
ization. Of note, none of the adherence studies cited exclusively examined the newer tools in samples of older adults, so further research about the acceptability of emerging solutions for older adults is needed.

\section{Conclusions}

The transitions following hospitalization that older adults experience present challenges to medication adherence. As they are coping with changes to their health, older adults frequently must also manage multiple medication changes. The risk of nonadherence is high if medication discrepancies are not resolved. When older adults pass through the settings in the continuum of care, health care providers have multiple opportunities to assess the older adult's degree of adherence, inquire about reasons for nonadherence and recommend tools to aid adherence. The evidence to support which tools are most appropriate and most effective is still evolving. However, care transitions represent touch points at which health care providers can review all medications and then assess and foster adherence to the medication regimen. Through these steps, the safety of older adults will be optimized following hospitalization.

\section{Disclosure Statement}

Dr. Mixon is supported by a VA HSR\&D Career Development Award (12-168) and in part by Veterans Affairs Clinical Research Center of Excellence and the Geriatric Research, Education and Clinical Center. The views expressed in this article are those of the author(s) and do not necessarily represent the views of the Department of Veterans Affairs. Dr. Bell is supported by an NICHD Mentored Clinical Scientist Development Award (2K12HD043483-11, P.I. Hartmann). Dr. Kripalani is funded in part by AHRQ R18 HS019598, 1C1CMS331006-01-02 and 1C1CMS330979-01. He is a consultant to and holds equity in PictureRx, LLC. Dr. Neal has served as a consultant for PictureRx, LLC. Dr. Powers is funded by HRSA Geriatric Education Centers Grant 1D31HP08823-01-00 and serves on the Pharmacy Advisory Committee for HealthSpring.

\section{References}

1 Wilson IB, Schoen C, Neuman P, Strollo MK, Rogers WH, Chang H, Safran DG: Physicianpatient communication about prescription medication nonadherence: a 50-state study of America's seniors. J Gen Intern Med 2007;22: 6-12.

2 Garcia-Caballos M, Ramos-Diaz F, JimenezMoleon JJ, Bueno-Cavanillas A: Drug-related problems in older people after hospital discharge and interventions to reduce them. Age Ageing 2010;39:430-438.

3 Fallis BA, Dhalla IA, Klemensberg J, Bell CM: Primary medication non-adherence after discharge from a general internal medicine service. PLoS One 2013;8:e61735.

4 Coleman EA, Smith JD, Raha D, Min SJ: Posthospital medication discrepancies: prevalence and contributing factors. Arch Intern Med 2005;165:1842-1847.

5 Ma E, Coleman EA, Fish R, Lin M, Kramer AM: Quantifying posthospital care transitions in older patients. J Am Med Dir Assoc 2004;5:71-74.

6 The Joint Commission: National patient safety goals, effective January 1, 2013. Hospital Accreditation Program. Chicago, The Joint Commission, 2012, pp 1-16.

7 Harris CM, Sridharan A, Landis R, Howell E, Wright S: What happens to the medication regimens of older adults during and after an acute hospitalization? J Patient Saf 2013;9:150-153.

${ }_{8}$ Tjia J, Bonner A, Briesacher BA, McGee S, Terrill E, Miller K: Medication discrepancies upon hospital to skilled nursing facility transitions. J Gen Intern Med 2009;24:630635.

-9 Mor V, Intrator O, Feng Z, Grabowski DC The revolving door of rehospitalization from skilled nursing facilities. Health Aff (Millwood) 2010;29:57-64.

10 Wier LM, Andrews RM: Hospital utilization among oldest adults, 2008. HCUP Statistical Brief 103. Rockville, Agency for Healthcare Research and Quality, 2010.

-11 Corbett CF, Setter SM, Daratha KB, Neumiller JJ, Wood LD: Nurse-identified hospital to home medication discrepancies: implications for improving transitional care. Geriatr Nurs 2010;31:188-196.

12 National Center for Health Statistics: National Home and Hospice Care Survey. Fact sheet. Atlanta, Centers for Disease Control and Prevention, 2007.

13 Sokol MC, McGuigan KA, Verbrugge RR, Epstein RS: Impact of medication adherence on hospitalization risk and healthcare cost. Med Care 2005;43:521-530.

14 Col N, Fanale JE, Kronholm P: The role of medication noncompliance and adverse drug reactions in hospitalizations of the elderly. Arch Intern Med 1990;150:841-845.

15 Smith JD, Coleman EA, Min SJ: A new tool for identifying discrepancies in postacute medications for community-dwelling older adults. Am J Geriatr Pharmacother 2004;2: 141-147.

16 Maniaci MJ, Heckman MG, Dawson NL: Functional health literacy and understanding of medications at discharge. Mayo Clin Proc 2008;83:554-558.

17 Mudge AM, O’Rourke P, Denaro CP: Timing and risk factors for functional changes associated with medical hospitalization in older patients. J Gerontol A Biol Sci Med Sci 2010;65: 866-872.

18 Desai M, Pratt LA, Lentzner H, Robinson KN: Trends in vision and hearing among older Americans. Aging Trends, No 2. Hyattsville, National Center for Health Statistics, 2001.

19 Morbidity and Mortality Weekly Report: Prevalence of doctor-diagnosed arthritis and arthritis-attributable activity limitation United States, 2007-2009. Atlanta, Centers for Disease Control and Prevention, 2010, vol 59, pp 1261-1265.

20 Lindquist LA, Go L, Fleisher J, Jain N, Baker D: Improvements in cognition following hospital discharge of community dwelling seniors. J Gen Intern Med 2011;26:765-770.

21 Kvavilashvili L, Kornbrot DE, Mash V, Cockburn J, Milne A: Differential effects of age on prospective and retrospective memory tasks in young, young-old, and old-old adults. Memory 2009; 17:180-196.

22 Kvavilashvili L, Cockburn J, Kornbrot DE: Prospective memory and ageing paradox with event-based tasks: a study of young, youngold, and old-old participants. Q J Exp Psychol (Hove) 2013;66:864-875.

23 Institute of Medicine: Health Literacy. A Prescription to End Confusion. Washington, National Academies Press, 2004. 
24 Kutner M, Greenberg E, Jin Y, Paulsen C: The health literacy of America's adults: Results from the 2003 National Assessment of Adult Literacy (NCES 2006-483). Washington, US Department of Education, National Center for Education Statistics, 2006.

25 Hilmer S, Ford G: Hazzard's Geriatric Medicine and Gerontology, ed 6. New York, McGraw-Hill, 2009.

26 Mühlberg W, Platt D: Age-dependent changes of the kidneys: pharmacological implications. Gerontology 1999;45:243-253.

27 Le Couteur DG, McLean AJ: The aging liver. Drug clearance and an oxygen diffusion barrier hypothesis. Clin Pharmacokinet 1998;34: 359-373.

-28 American Geriatrics Society updated Beers Criteria for potentially inappropriate medication use in older adults. J Am Geriatr Soc 2012;60:616-631.

29 McLean AJ, Le Couteur DG: Aging biology and geriatric clinical pharmacology. Pharmacol Rev 2004;56:163-184.

- 30 Shaffer D, Singer S, Korvick J, Honig P: Concomitant risk factors in reports of torsades de pointes associated with macrolide use: review of the United States Food and Drug Administration Adverse Event Reporting System. Clin Infect Dis 2002;35:197-200.

31 Fox C, Richardson K, Maidment ID, Savva GM, Matthews FE, Smithard D, Coulton S, Katona C, Boustani MA, Brayne C: Anticholinergic medication use and cognitive impairment in the older population: the Medical Research Council Cognitive Function and Ageing study. J Am Geriatr Soc 2011;59:1477-1483.

- 32 Soumerai SB, Pierre-Jacques M, Zhang F, Ross-Degnan D, Adams AS, Gurwitz J, Adler G, Safran DG: Cost-related medication nonadherence among elderly and disabled Medicare beneficiaries: a national survey 1 year before the Medicare drug benefit. Arch Intern Med 2006;166:1829-1835.

33 Administration on Aging, Living AfC: A profile of older Americans: 2012, USA. Washington, Department of Health and Human Services, 2012.

-34 MacLaughlin EJ, Raehl CL, Treadway AK, Sterling TL, Zoller DP, Bond CA: Assessing medication adherence in the elderly: which tools to use in clinical practice? Drugs Aging 2005;22:231-255.

35 Li P, McElligott S, Bergquist H, Schwartz JS, Doshi JA: Effect of the Medicare Part D coverage gap on medication use among patients with hypertension and hyperlipidemia. Ann Intern Med 2012;156:776-784, W-263, W-264, W-265, W-266, W-267, W-268, W-269.
36 Choudhry NK, Avorn J, Glynn RJ, Antman EM, Schneeweiss S, Toscano M, Reisman L, Fernandes J, Spettell C, Lee JL, Levin R, Brennan T, Shrank WH: Full coverage for preventive medications after myocardial infarction. N Engl J Med 2011;365:2088-2097.

37 Hirth RA, Greer SL, Albert JM, Young EW, Piette JD: Out-of-pocket spending and medication adherence among dialysis patients in twelve countries. Health Aff (Millwood) 2008; 27:89-102.

- 38 Morandi A, Vasilevskis E, Pandharipande PP, Girard TD, Solberg LM, Neal EB, Koestner T, Torres RE, Thompson JL, Shintani AK, Han JH, Schnelle JF, Fick DM, Ely EW, Kripalani $S$ : Inappropriate medication prescriptions in elderly adults surviving an intensive care unit hospitalization. J Am Geriatr Soc 2013;61: 1128-1134.

39 Barry PJ, Gallagher P, Ryan C, O’Mahony D: START (screening tool to alert doctors to the right treatment) - an evidence-based screening tool to detect prescribing omissions in elderly patients. Age Ageing 2007;36:632-638.

40 Levy HB, Marcus EL, Christen C: Beyond the Beers Criteria: a comparative overview of explicit criteria. Ann Pharmacother 2010;44: 1968-1975.

41 O'Mahony D, Gallagher P, Ryan C, Byrne S, Hamilton H, Barry P, O'Connor M, Kennedy J: STOPP and START criteria: a new approach to detecting potentially inappropriate prescribing in old age. Eur Geriatr Med 2010; $1: 45-51$.

42 Patient-centered care for older adults with multiple chronic conditions: a stepwise approach from the American Geriatrics Society: American Geriatrics Society Expert Panel on the Care of Older Adults with Multimorbidity. J Am Geriatr Soc 2012;60:1957-1968.

43 Claxton AJ, Cramer J, Pierce C: A systematic review of the associations between dose regimens and medication compliance. Clin Ther 2001;23:1296-1310.

44 Steiner JF, Prochaska AV: The assessment of refill compliance using pharmacy records: methods, validity, and applications. J Clin Epidemiol 1997;50:105-116.

45 Edelberg HK, Shallenberger E, Wei JY: Medication management capacity in highly functioning community-living older adults: detection of early deficits. J Am Geriatr Soc 1999; 47:592-596.
46 Raehl CL, Bond CA, Woods T, Patry RA, Sleeper RB: Individualized drug use assessment in the elderly. Pharmacotherapy 2002; 22:1239-1248.

47 Kripalani S, Risser J, Gatti M, Jacobson TA: Development and evaluation of the Adherence to Refills and Medications Scale (ARMS) among low-literacy patients with chronic disease. Value Health 2009;12:118-123.

48 Shi L, Liu J, Fonseca V, Walker P, Kalsekar A, Pawaskar M: Correlation between adherence rates measured by MEMS and self-reported questionnaires: a meta-analysis. Health Qual Life Outcomes 2010;8:99.

$49 \mathrm{Wu}$ JR, Dewalt DA, Baker DW, Schillinger D, Ruo B, Bibbins-Domingo K, MacabascoO'Connell A, Holmes GM, Broucksou KA, Erman B, Hawk V, Cene CW, Jones CD, Pignone $\mathrm{M}$ : A single-item self-report medication adherence question predicts hospitalisation and death in patients with heart failure. J Clin Nurs 2014;23:2554-2564.

50 Kripalani S, Yao X, Haynes RB: Interventions to enhance medication adherence in chronic medical conditions: a systematic review. Arch Intern Med 2007;167:540-550.

-51 Katz MG, Kripalani S, Weiss BD: Use of pictorial aids in medication instructions: a review of the literature. Am J Health Syst Pharm 2006;63:2391-2397.

52 Vervloet M, Linn AJ, van Weert JC, de Bakker DH, Bouvy ML, van Dijk L: The effectiveness of interventions using electronic reminders to improve adherence to chronic medication: a systematic review of the literature. J Am Med Inform Assoc 2012;19:696-704.

53 de Jongh T, Gurol-Urganci I, VodopivecJamsek V, Car J, Atun R: Mobile phone messaging for facilitating self-management of long-term illnesses. Cochrane Database Syst Rev 2012;12:CD007459.

54 Hughes LD, Done J, Young A: Not 2 old 2 TXT: there is potential to use email and SMS text message healthcare reminders for rheumatology patients up to 65 years old. Health Informatics J 2011;17:266-276.

55 Ruppar TM, Conn VS, Russell CL: Medication adherence interventions for older adults: literature review. Res Theory Nurs Pract 2008; 22:114-147.

-56 Zedler BK, Kakad P, Colilla S, Murrelle L, Shah NR: Does packaging with a calendar feature improve adherence to self-administered medication for long-term use? A systematic review. Clin Ther 2011;33:62-73.

57 National Health Service West Midlands: The Automated Pill Dispenser Project - end project evaluation report. London, National Health Service, 2012 